\title{
Linee guida per la sorveglianza della densità minerale ossea in bambini, adolescenti e giovani adulti sopravvissuti a cancro: raccomandazioni evidence-based di un gruppo di esperti
}

\author{
Giovanna Mantovani $^{1} \cdot$ Alessandra Mangone $^{1}$
}

Accettato: 15 novembre 2021 / Pubblicato online: 21 gennaio 2022

(c) The Author(s) 2022

Commento a:

Bone mineral density surveillance for childhood, adolescent, and young adult cancer survivors: evidence-based recommendations from the International Late Effects of Childhood Cancer Guideline Harmonization Group.

J.E. van Atteveld, R.L. Mulder,

M.M. van den Heuvel-Eibrink, M.M. Hudson, L.C. Kremer, R. Skinner, W.H. Wallace, L.S. Constine, C.E. Higham, S.C. Kaste, R. Niinimäki,

S. Mostoufi-Moab, N. Alos, D. Fintini, K.J. Templeton, L.M. Ward, E. Frey, R. Franceschi, V. Pavasovic, S.E. Karol, N.L. Amin, L.M. Vrooman, A. Harila-Saari, C. Demoor-Goldschmidt, R.D. Murray, E. Bardi, M.H. Lequin, M.F. Faienza, O. Zaikova, C. Berger, S. Mora, K.K. Ness, S.J. Neggers, S.M. Pluijm, J.H. Simmons, N. Di Iorgi.

Lancet Diabetes Endocrinol (2021) 9(9):622-637

La popolazione pediatrica sopravvissuta a neoplasie presenta un aumentato rischio di andare incontro a ridotta mineralizzazione scheletrica per mancato raggiungimento del picco di massa ossea, che normalmente avviene intorno ai 20-30 anni, con frequente riscontro di Bone Mineral Density (BMD) ridotta rispetto ai coetanei e conseguente aumentato rischio fratturativo. Questa condizione può essere dovuta sia alla malattia di base, sia ai trattamenti antineoplastici ricevuti, sia a una serie di possibili fattori di rischio addizionali che risultano di più comune riscontro in tale popolazione, tra cui difetti endocrini, malnutrizione e vita sedentaria [1]. Le linee guida attuali, pur riconoscendo l'importanza di

G. Mantovani

giovanna.mantovani@unimi.it

1 Unità di Endocrinologia, Fondazione IRCCS Ca' Granda Ospedale Maggiore Policlinico, Dipartimento di Scienze Cliniche e di Comunità, Università degli Studi di Milano, Milano, Italia una diagnosi e trattamento precoce, mostrano delle discordanze in merito. Per questo motivo, un gruppo di 36 esperti da 10 paesi (International Late Effects of Childhood Cancer Guideline Harmonization Group) ha stilato, a seguito di un'analisi critica e integrata della letteratura, una serie di pratiche raccomandazioni evidence-based che di seguito vengono riassunte, coi relativi gradi di raccomandazione a seconda della qualità delle evidenze riscontrate [2].

- I bambini, gli adolescenti e i giovani adulti sopravvissuti a neoplasie e i loro medici di riferimento dovrebbero essere consapevoli del rischio di presentare una massa ossea ridotta (Z-score $\leq-1$ e $>-2$ ) o molto ridotta (Z-score $\leq-2$ ) e prestare attenzione alle possibili conseguenze che ne derivano come lombalgia, fratture a seguito di traumi minori e perdita di altezza conseguente a deformazioni vertebrali (raccomandazione forte). I principali fattori di rischio legati al trattamento ricevuto comprendono, in particolare, radioterapia craniale/craniospinale (alta qualità di evidenza per BMD molto ridotta), irradiazione total-body (alta qualità di evidenza per BMD ridotta), e uso di corticosteroidi come terapia antineoplastica (moderata qualità di evidenza per BMD ridotta), sebbene non sia stato possibile determinarne dei valori soglia. Altri fattori di rischio per ridotta mineralizzazione ossea che sono stati osservati in questa popolazione includono ipogonadismo e deficit di ormone della crescita, spesso per effetto delle terapie ricevute, ridotto BMI, sesso maschile, razza bianca, ridotta attività fisica e abitudine tabagica.

- Lo screening della BMD è raccomandato per tutti i bambini, adolescenti e giovani adulti cancer-survivors sottoposti a radioterapia craniale o craniospinale (raccomandazione forte). Tale screening è ragionevole anche per coloro che sono stati trattati con irradiazione total-body (raccomandazione moderata). A causa di evidenze insufficienti, non può essere formulata una raccomandazione relativa alla sorveglianza della densità scheletrica per la popolazione pediatrica trattata con corticosteroidi a sco- 
po antineoplastico. In quest'ultima categoria, bisognerebbe operare una valutazione personalizzata considerando gli eventuali fattori di rischio addizionali del paziente e tutti i possibili benefici ed effetti collaterali del caso.

- Per lo screening di massa ossea si raccomanda l'utilizzo del metodo di scansione densitometrica (DXA) a livello del rachide lombare e/o total body less head (TBLH) per bambini e adolescenti e femore totale per adolescenti e giovani adulti (raccomandazione forte). La TC quantitativa non è raccomandata come metodica di sorveglianza.

- Lo screening è raccomandato con l'inizio del follow-up a lungo termine del paziente sopravvissuto a tumore, ovvero tra 2 e 5 anni dopo il termine delle terapie oncologiche. Se la BMD di screening risulta normale (Z-score $>-1$ ) è consigliato ripetere la DXA a 25 anni di età, ovvero quando dovrebbe essere raggiunto il picco di massa ossea (raccomandazione forte).

- In caso di riscontro di Z-score $\leq-2$ è raccomandato rivolgersi a uno specialista dell'osso per eseguire ulteriori valutazioni, per la corretta interpretazione dei dati e per l'eventuale impostazione di una terapia specifica e del relativo follow-up (raccomandazione forte).

- In caso di Z-score rilevato $\leq-1$ e $>-2$, si raccomanda innanzitutto di indagare la presenza di eventuali difetti endocrinologici, in particolare ipogonadismo e deficit di $\mathrm{GH}$, quindi di consultare uno specialista per valutare ulteriori indagini. Ripetere la DXA dopo 2 anni e, quindi, come clinicamente indicato a seconda del trend della BMD (raccomandazione forte).

- In tutti i casi è raccomandato, a prescindere dalla densità minerale riscontrata, approfondire le abitudini del paziente e incoraggiare uno stile di vita che promuova la salute dell'osso, ovvero: praticare attività fisica, astenersi dall'abitudine tabagica, limitare/evitare l'assunzione di alcol, apportare un adeguato introito di vitamina D (almeno $400 \mathrm{IU} / \mathrm{die}$ ) e calcio (almeno $500 \mathrm{mg} / \mathrm{die}$ ) e, eventualmente, supplementare secondo le linee guida nazionali, adeguare lo stato nutrizionale in caso di BMI ridotto (raccomandazione forte). Tali misure risultano, infatti, più efficaci durante la pubertà, in particolare l'esercizio fisico e la terapia ormonale sostitutiva, e il picco di massa ossea raggiunto risulta predittivo dello stato futuro della salute scheletrica.

- È ragionevole, infine, indirizzare i pazienti a uno specialista dell'osso qualora siano già presenti o si riscontrino nel corso del follow-up fratture (vertebrali e non) a seguito di traumi a basso impatto (raccomandazione moderata).

Open Access This article is licensed under a Creative Commons Attribution 4.0 International License, which permits use, sharing, adaptation, distribution and reproduction in any medium or format, as long as you give appropriate credit to the original author(s) and the source, provide a link to the Creative Commons licence, and indicate if changes were made. The images or other third party material in this article are included in the article's Creative Commons licence, unless indicated otherwise in a credit line to the material. If material is not included in the article's Creative Commons licence and your intended use is not permitted by statutory regulation or exceeds the permitted use, you will need to obtain permission directly from the copyright holder. To view a copy of this licence, visit http://creativecommons.org/licenses/by/4.0/.

\section{Bibliografia}

1. van Iersel L, Li Z, Srivastava DK et al (2019) Hypothalamicpituitary disorders in childhood cancer survivors: prevalence, risk factors and long-term health outcomes. J Clin Endocrinol Metab 104(12):6101-6115

2. van Atteveld JE, Mulder RL, van den Heuvel-Eibrink MM et al (2021) Bone mineral density surveillance for childhood, adolescent, and young adult cancer survivors: evidence-based recommendations from the International Late Effects of Childhood Cancer Guideline Harmonization Group. Lancet Diabetes Endocrinol 9(9):622-637

Nota della casa editrice Springer Nature rimane neutrale in riguardo alle rivendicazioni giurisdizionali nelle mappe pubblicate e nelle affiliazioni istituzionali. 